\title{
Participación social y educación ambiental para la conservación. Un estudio de caso con niños y jóvenes de una zona rural periurbana.
}

\author{
Víctor Ávila-Akerberg* \\ Tanya González-Martínez \\ Instituto de Ciencias Agropecuarias y Rurales \\ Universidad Autónoma del Estado de México
}

\section{Resumen}

No obstante la trascendencia de la riqueza biológica de sus bosques, México sigue perdiendo superficie forestal año con año, a pesar de brindar gran cantidad de servicios ambientales. Los bosques que rodean la Ciudad de México enfrentan una presión antrópica muy fuerte a causa de las necesidades de más de 21 millones de habitantes. La sobreexplotación de los mantos acuíferos, el cambio de uso de suelo, la desaparición de los ríos y la contaminación del aire son solo algunos de los problemas ambientales más comunes. Las áreas boscosas han quedado reducidas a las zonas montañosas más altas, muchas de ellas habitadas por asentamientos rurales antiguos, originalmente pequeños y aislados, pero que hoy crecen con rapidez y demandan servicios urbanos. Mediante talleres de educación ambiental en dos municipios semirrurales al noroeste de la Ciudad de México se tuvo un acercamiento con 855 niños y jóvenes en 2013, 1060 en 2014 y 1770 en 2015, de escuelas de nivel básico y medio (de 9 a 18 años de edad), para transferir investigación académica y conocer sus percepciones sobre la biodiversidad y la problemática ambiental local. Se organizaron además concursos de carteles donde mostraron la relevancia biológica y ambiental de los bosques en su comunidad. De manera intencional, los carteles concursantes fueron evaluados por autoridades, con lo que se consiguió enfrentar, bajo esta temática, a los niños y jóvenes con tomadores de decisiones. En general, los primeros reconocieron muchos elementos de la biodiversidad y mencionaron que lo que más les gusta de su comunidad es la naturaleza, que es importante no tirar los árboles y mantener limpios el bosque y sus ríos.

\section{Palabras clave}

Servicios ecosistémicos, biodiversidad, megaciudades, educación básica y media, educación ambiental. 


\title{
Conservation through social involvement and environmental education. A case study with children and young people in a rural peri-urban area.
}

\author{
Víctor Ávila-Akerberg* \\ Tanya González-Martínez \\ Instituto de Ciencias Agropecuarias y Rurales \\ Universidad Autónoma del Estado de México
}

\begin{abstract}
Not withstanding the relevance of the biological wealth of its forest, Mexico continues to loose forest areas. Forests that surround Mexico City face a strong anthropic pressure due to the needs of more than 21 million inhabitants. Forest areas have been reduced to the higher mountain zones, often inhabited by ancient rural settlements, originally small and isolated but at present growing rapidly and demanding urban services. In order to transfer academic research and to appreciate their perception on biodiversity and local environmental problems, environmental education workshops were conducted in two semirural municipalities located northwest of Mexico City. 855 children and youngsters were approached in 2013, 1060 in 2014 and 1770 in 2015, all of them students at primary and secondary level ( 9 to 18 years of age). In addition, poster contests were organized on the biological and environmental relevance of the community forests. These posters were evaluated by local authorities, which allowed children and youngsters to interact with decision-makers. In general children recognized many elements of their community biodiversity and mentioned that what they like most is nature, and that it is important not to cut trees and keep forests and rivers clean.
\end{abstract}

Key words

Ecosystem services, biodiversity, megacities, primary and secondary education, environmental education. 


\section{Introducción}

El desarrollo socioambiental mundial, vinculado con una evolución en la relación sociedad-naturaleza alrededor de los procesos productivos, ha llevado a una disminución de la calidad de vida de diversos sectores de la sociedad humana y a un deterioro ambiental generalizado. Los problemas ambientales ocurren a diferentes escalas y es cada vez más difícil distinguir entre fuentes y afectados. Estos problemas han provocado una crisis ambiental debida principalmente a factores socioeconómicos, políticos y culturales. Parte de su solución debe estar fundamentada en una mayor participación social con conocimiento real en torno a los efectos de la interacción entre la naturaleza y las actividades humanas. En la conservación de la naturaleza, dos de los elementos que más incidencia pueden tener sobre las generaciones más jóvenes es la educación ambiental y la participación social.

Hablar de educación ambiental con frecuencia se refiere a "la necesidad de informar a las personas acerca de las características del medio en el que ellas actúan [...] y el efecto que [...] dichas acciones tienen sobre el ambiente" (Sarukhán, 2006: vii). Partiendo de esta idea, en el presente artículo se reportan esfuerzos para socializar información a grupos locales sobre biodiversidad, servicios ambientales y problemáticas del entorno natural en el que habitan, y cómo su propio comportamiento puede mejorar o disminuir su calidad. Además se buscó la participación social de niños y jóvenes estudiantes y sus familias, el gobierno local y algunos actores clave en el proceso de toma de decisiones. Esta investigación muestra una experiencia en educación ambiental con base en la transferencia de información científica y en la promoción de la participación social en dos municipios semirrurales ubicados en la zona montañosa adyacente a la Ciudad de México. La región es proveedora importante de servicios ambientales fundamentales como agua y aire limpios, pero con un riesgo constante de desaparecer por contaminación y cambios de uso de suelo.

\section{Antecedentes}

De acuerdo con la revisión histórica descrita por Bravo Mercado et al. (2008), la educación ambiental en México se ha ido consolidando con los años. Las primeras labores en ese ámbito se centraban en actividades organizadas por la 
Participación social y educación ambiental para la conservación. Un estudio de caso con niños y jóvenes de una zona rural periurbana

sociedad civil que tomaban como base el legado histórico de la interpretación de los pueblos indígenas acerca de la relación entre las comunidades humanas y su entorno. No fue sino hasta la década de 1940 cuando la academia mexicana empezó a generar "propuestas educativas relacionadas con el conocimiento de la biología y la importancia de la conservación de animales y plantas” (Barahona y Almeida-Leñero, 2008). Durante los ochenta, la Secretaría de Desarrollo Urbano y Ecología (Sedue) formalizó acciones educativas concretas vinculadas con el ambiente y promovió la revisión de contenidos ambientales en los programas y libros de texto -desde preescolar hasta bachillerato y normal- de la Secretaría de Educación Pública (SEP), época en que se construyó el marco conceptual de lo que sería la educación ambiental en nuestro país. En los noventa ya se organizaban eventos académicos para dar capacitaciones en campo e intercambiar experiencias y surgían redes de educadores ambientales entre grupos ecologistas, académicos y de funcionarios, con redes regionales y estatales de educadores ambientales. Numerosas actividades como la Primera Reunión Nacional de Educadores Ambientales (Morelos, 1992), el Primer Congreso Iberoamericano de Educación Ambiental (Guadalajara, 1992), la 23 ${ }^{\text {a }}$ Reunión Anual de la North American Association for Environmental Education (Cancún, 1994), o las convenciones internacionales sobre Medio Ambiente y Desarrollo (Aguascalientes, 1999), por mencionar algunas, sirvieron para discutir acciones estratégicas en materia de educación ambiental y organizar a los educadores en redes regionales (Camarena-Gómez, 2006) que hoy constituyen la Red de Centros de Educación Ambiental.

Para la primera década del siglo xxi, los planes de los 31 estados mexicanos incluían ya acciones concretas de capacitación y comunicación educativa para el desarrollo sustentable. En la actualidad, siguiendo la línea de la Organización de las Naciones Unidas para la Educación, la Ciencia y la Cultura (Unesco), el concepto de educación ambiental se ha orientado hacia la sustentabilidad como una forma de vida. Así, en México existe el Centro de Educación y Capacitación para el Desarrollo Sustentable (Cecadesu) de la Secretaría de Medio Ambiente y Recursos Naturales (Semarnat), el cual ofrece múltiples posibilidades para fortalecer la educación ambiental y apoyar la legislación local por medio de la Ley General del Equilibrio Ecológico y la Protección al Ambiente (LGEEPA). 
Sin embargo, a pesar de todos estos programas, se han aplicado muy pocos cambios en la educación ambiental, enfocándose principalmente en temas de índole global (cambio climático, deforestación, pérdida de biodiversidad, etc.). Asimismo, el notable deterioro del ambiente debido a causas antropogénicas y la sobreexplotación de los recursos naturales para el desarrollo de la sociedad humana han reavivado el interés en la educación ambiental (Vega y Álvarez, 2005) como una herramienta para adquirir conocimientos, interiorizar actitudes, despertar la conciencia crítica y cambiar conductas individuales y colectivas (Bravo Mercado et al., 2008) en los niveles más cercanos a la realidad de la vida diaria.

El gran reto de la educación ambiental es lograr que las personas comprendan que las acciones individuales pueden contribuir, remediar o prevenir el daño ambiental. Es fundamental transmitir a las nuevas generaciones que la forma en la que usamos y manejamos los recursos naturales incide en el legado que dejaremos a las generaciones venideras. La educación ambiental pretende recuperar el proceso que lleva a entender y apreciar la relación mutua entre el hombre, su cultura y el ambiente natural, esperando que esta revalorización se refleje en un aprovechamiento racional de los recursos naturales, con una visión que reconozca el valor intrínseco del sistema natural completo, más allá de la perspectiva utilitaria.

Dada la noción de que normalmente es menos complejo enseñar a un niño o joven que hacer cambiar de ideas a un adulto, fue este grupo el que se tomó como base para una serie de pláticas de educación ambiental impartidas a niños y jóvenes de niveles de educación básico y medio en dos municipios semirrurales (baja densidad de población y presencia de actividades agropecuarias) al noroeste de la Ciudad de México: Isidro Fabela y Jilotzingo. Se buscó promover la participación e integración al cuidado de su ambiente, reforzando su identidad con la naturaleza y biodiversidad característica de la región, y la relevancia de los ecosistemas en la provisión de bienes y servicios ambientales, tanto para los municipios como para la cuenca hidrológica a la que pertenecen y el resto de la Zona Metropolitana de la Ciudad de México. Mediante el acercamiento con los niños y jóvenes se pretendió conocer la percepción que tienen sobre su entorno y las alternativas para su mejor uso y cuidado con el objetivo de fomentar su participación y la transferencia de información. Un concurso 
Participación social y educación ambiental para la conservación. Un estudio de caso con niños y jóvenes de una zona rural periurbana

anual de carteles sirvió como herramienta de motivación y al mismo tiempo como enseñanza para aprender haciendo. Además, se buscó la confrontación de los niños y jóvenes con el gobierno municipal y otras autoridades, lo que expuso el manejo que tiene un gobierno ante su territorio.

El cambio en la interacción humano-ambiente no se basa exclusivamente en los niños y jóvenes, pero se cree que es el sector poblacional más importante, ya que ellos serán los que dirijan la toma de decisiones hacia el uso y cuidado de su entorno en un futuro.

\section{Metodología}

La Sierra de las Cruces es la cordillera que separa los valles de las ciudades de México y Toluca. En la sección norte de la sierra, mirando hacia la Ciudad de México, se extiende la subcuenca de la presa Guadalupe, de una superficie aproximada de 38000 hectáreas (Conagua y сCPG, 2008). La configuran cinco municipios del Estado de México: Atizapán de Zaragoza (con 476000 habitantes), Cuautitlán Izcalli (498 000), Nicolás Romero (366 000), Jilotzingo (18 000) e Isidro Fabela (14 000). En la parte más alta de la cuenca se encuentra el corredor biológico Otomí-Mexica, área natural protegida en el nivel estatal.

La conforman montañas y cañadas con bosques de oyamel (Abies religiosa), pinos (Pinus hartwegii, P. montezumae, P. patula) y encinos (Quercus rugosa, Q. laurina, etc.). La precipitación media es de $953 \mathrm{~mm}$ bajo un gradiente altitudinal que va de los 3750 a los $2200 \mathrm{msnm}$. La zona alta (>3 $000 \mathrm{msnm}$ ) constituye un área importante de recarga natural del acuífero de la Ciudad de México. Se caracteriza por una baja presencia de asentamientos humanos, alta cobertura de bosques y manantiales con agua de gran calidad, lo que permite el desarrollo de la actividad agrícola y acuícola. La densidad poblacional aumenta progresivamente hacia la zona media (3 000-2 $500 \mathrm{msnm}$ ), donde predominan las actividades agrícolas y pecuarias, así como el cambio de uso de suelo forestal a agrícola y habitacional. La parte baja ( $<2500 \mathrm{msnm}$ ) se distingue por estar totalmente cubierta por asentamientos urbanos de alta densidad, poco organizados, y abarca la mitad de Nicolás Romero y todo el territorio de los municipios de Atizapán y Cuautitlán Izcalli. En esta zona se ubica la presa Guadalupe, cuyo embalse comúnmente se conoce como Lago de Guadalupe. 


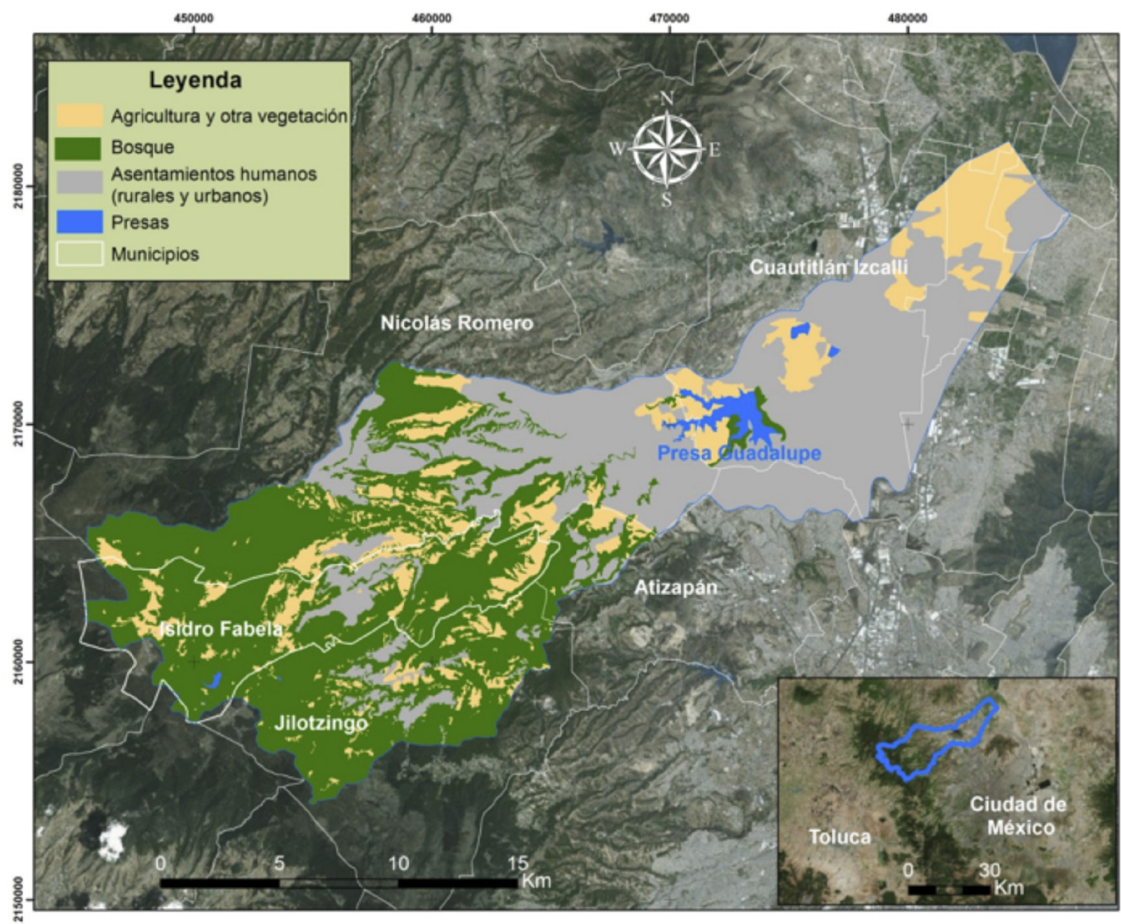

FIgURA 1. Localización de la cuenca presa Guadalupe (en azul) y los municipios de Isidro Fabela y Jilotzingo, al noroeste de la Ciudad de México

En esta experiencia se buscó un acercamiento con niños y jóvenes estudiantes de las escuelas públicas en los municipios de Isidro Fabela -cuya cabecera municipal es el poblado de Tlazala- y Jilotzingo -con cinco pueblos principales-, entidades con la menor densidad poblacional y mayor actividad agropecuaria de las cinco que conforman la cuenca presa Guadalupe. Durante tres años consecutivos (2013-2015) en Isidro Fabela se impartieron charlas de educación ambiental a niños y jóvenes estudiantes de escuelas públicas, a través de la gestión con el ayuntamiento de los permisos correspondientes para el trabajo con todos los niños de los grupos de las escuelas de educación básica y media. En 2015, esta labor se extendió al municipio vecino de Jilotzingo. Las escuelas y su ubicación se muestran en la figura 2, las cuales fueron: 
Participación social y educación ambiental para la conservación. Un estudio de caso con niños y jóvenes de una zona rural periurbana

- $4^{\circ}, 5^{\circ}$ y $6^{\circ}$ de las primarias Miguel Hidalgo, Tierra y Libertad, Benito Juárez, Las Palomas y Jarros (Isidro Fabela); Francisco I. Madero, Miguel Hidalgo, Benito Juárez e Ignacio Zaragoza (Jilotzingo).

- $1^{\circ}, 2^{\circ}$ y $3^{\circ}$ de las secundarias Justo Sierra y Técnica 73 (Isidro Fabela); Emilio Chuayffet, Manuel Tolsá y La Esperanza (Jilotzingo).

- $1^{\circ}, 2^{\circ}$ y $3^{\circ}$ del Centro de Bachillerato Tecnológico Isidro Fabela Alfaro (Isidro Fabela); Oficial No. 25 y Oficial No. 218 (Jilotzingo).

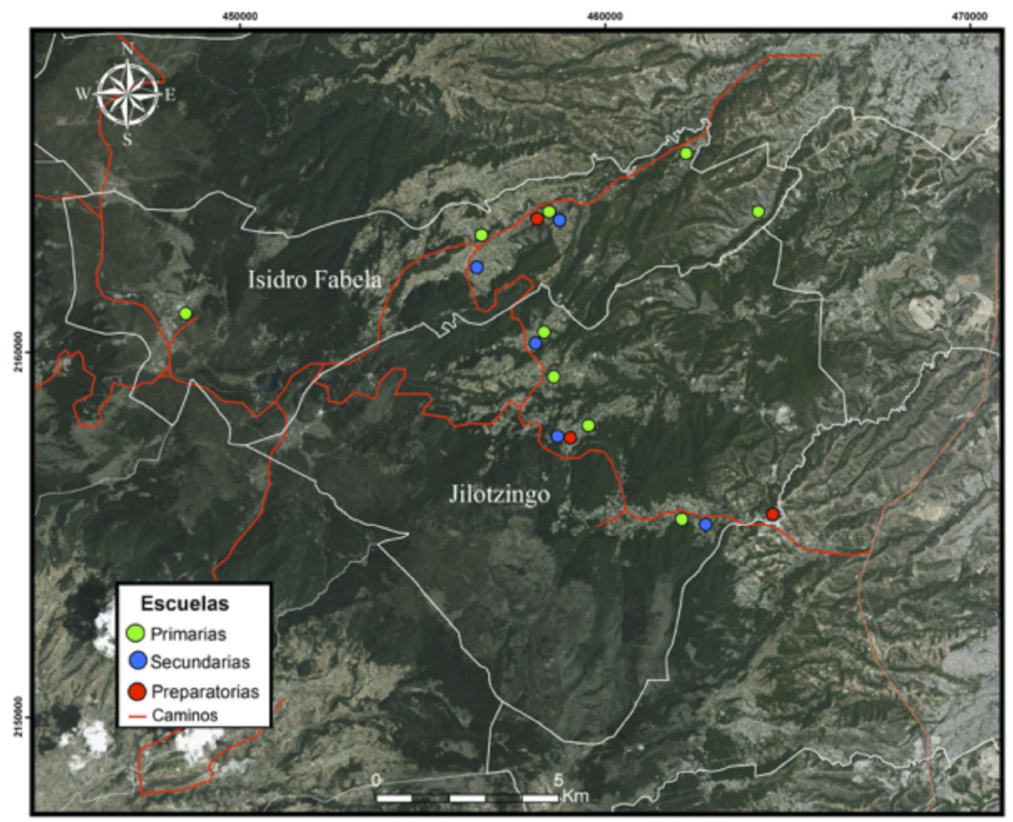

FiguRA 2. Ubicación de las escuelas de educación básica y media en Isidro Fabela y Jilotzingo

Antes de iniciar la charla, a todos los estudiantes se les pidió contestar un pequeño cuestionario con las siguientes preguntas:

- ¿Qué es lo que te gusta más y qué es lo que te gusta menos de tu municipio?

- ¿Cómo podrías ayudar en el buen uso y cuidado del monte, el agua, los animales, los árboles y las plantas?

- ¿Qué harías para resolver el problema de la basura?

- ¿Cómo te gustaría que fuera tu municipio en el futuro? 
Los datos recabados en los cuestionarios incluyeron algunos personales, como nombre -a fin de identificar a quienes ya habían recibido la plática el año anterior-, región donde habitan y lugar de origen, con objeto de advertir si hay una migración importante entre la población estudiantil. Estos datos han sido tratados y resguardados apropiadamente, con responsabilidad, y son de uso exclusivo para esta investigación.

Una vez contestado el cuestionario se comenzó la plática, con duración aproximada de una hora, a grupos de entre 35 y 70 estudiantes, con el apoyo de un proyector y una computadora. Se les habló sobre la ubicación de los municipios dentro de la cuenca presa Guadalupe, la biodiversidad y los recursos naturales disponibles en el municipio, haciendo énfasis en las especies de plantas, hongos y animales locales con las que los niños podrían sentirse más identificados. Finalmente se tocaron algunos problemas ambientales frecuentes, como las plagas del bosque, la tala ilegal, los incendios forestales, el abastecimiento de agua, la contaminación de los ríos y el manejo de la basura. Todas las charlas culminaban resaltando la belleza escénica de algunos parajes e invitando a los niños a participar en un concurso de carteles con la temática del cuidado y buen uso de los recursos naturales del área.

Para el concurso se hicieron tres categorías: primaria, secundaria y preparatoria, y en cada una hubo tres ganadores, a quienes se les otorgaron atractivos premios (ropa y balones deportivos, y la visita a un parque de diversiones) en un evento organizado con el apoyo de los ayuntamientos de Isidro Fabela y Jilotzingo y de algunos núcleos agrarios de la zona. Estos actores colaboraron tanto con la organización de la ceremonia de presentación y premiación de los carteles ganadores, como con el pago de los premios. La ceremonia siempre se realizó a principios de junio, aprovechando la celebración del Día Mundial del Medio Ambiente. Los jueces evaluadores fueron directores y maestros de las escuelas participantes, la presidenta municipal de Isidro Fabela, presidentes y representantes de bienes comunales y ejidales, un representante de la Comisión de Cuenca Presa Guadalupe, regidores de ambos municipios y autoridades ambientales estatales y nacionales (Protectora de Bosques del Estado de México -Probosque-, Semarnat, Comisión Nacional Forestal -Conafor-). El concurso se promocionó mediante carteles pegados en las escuelas y otros sitios concurridos, con las bases para participar (figura 3). 


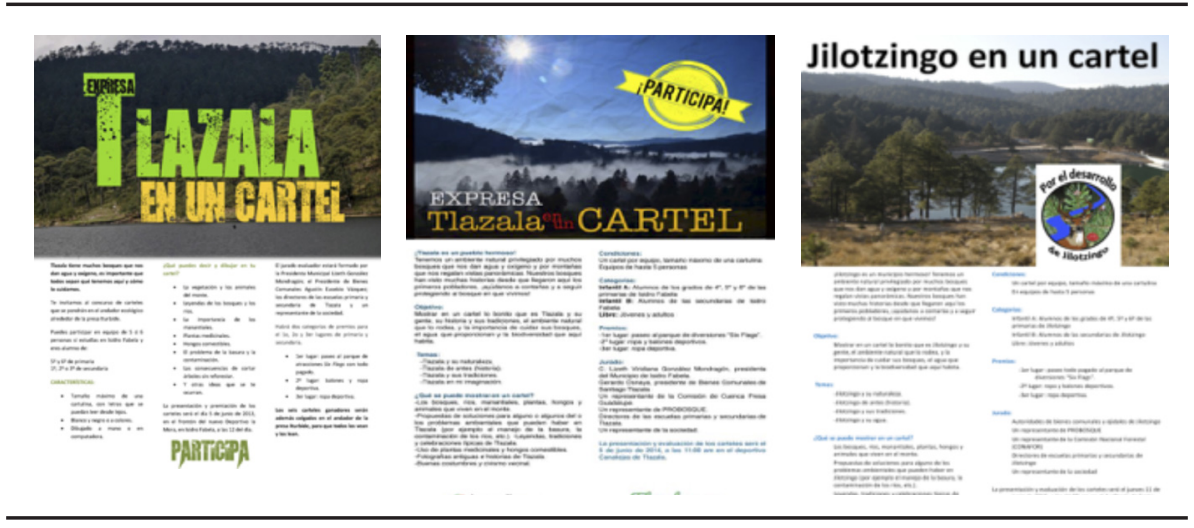

FigURA 3. Convocatoria para el concurso de carteles en Tlazala 2013 y 2014, y Jilotzingo 2015; se colocaron en las escuelas participantes y en espacios públicos

\section{Resultados y discusión}

Las pláticas se impartieron durante los últimos días de mayo a 855 niños y jóvenes en 2013, a 1060 en 2014 y a 1770 en 2015, en escuelas primarias, secundarias y preparatorias de los municipios de Isidro Fabela y Jilotzingo (figura 4). Si bien las instalaciones eran sencillas, no hubo mayor dificultad para efectuar las charlas, fuera de algunas fallas logísticas. Los maestros mostraron interés y disposición y algunos se emocionaron mucho al ver el material presentado y las reacciones de los alumnos. Los niños necesitaron entre diez y 15 minutos para contestar el cuestionario inicial, tras lo cual se comenzó con la charla de aproximadamente 50 minutos más.

El análisis del cuestionario supuso ciertas complicaciones metodológicas debido a que se trataba de preguntas abiertas con muchas posibilidades de respuesta, y se hizo así porque era una aproximación exploratoria y no se quiso influir de algún modo. Sin embargo, pronto nos dimos cuenta de que buena parte de las respuestas resultaban similares, por lo que a partir de ellas se crearon diversas categorías para cada una (figura 5). Por ejemplo, para la pregunta de lo que más les gustaba de su municipio, surgieron 18 categorías; la mayoría contestó que "la naturaleza", seguido de "la presa Iturbide", que es un embalse emblemático y bien conocido como atractivo turístico, ubicado en la parte alta 

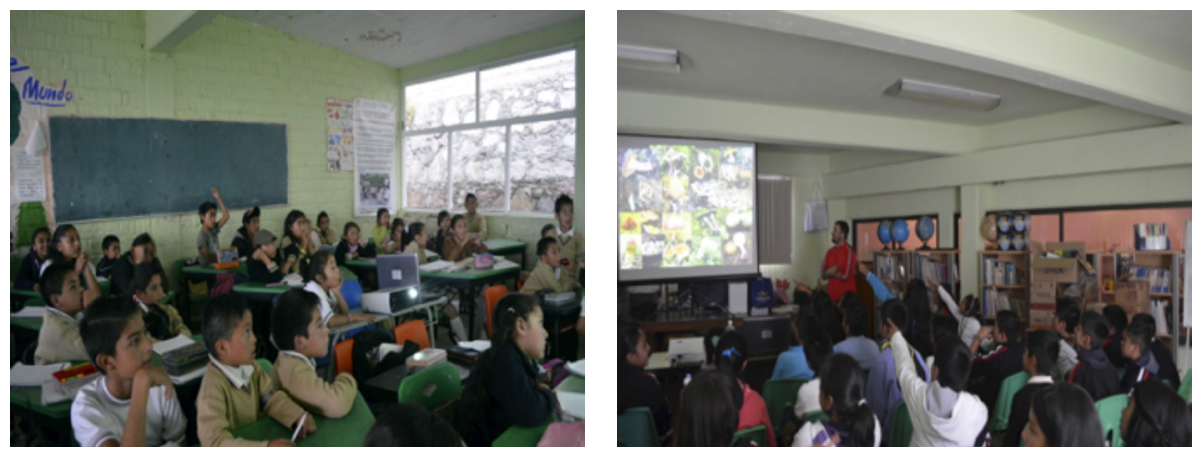

Figura 4. Pláticas de educación ambiental a niños y jóvenes entre 9 y 18 años en los municipios de Isidro Fabela y Jilotzingo, Estado de México

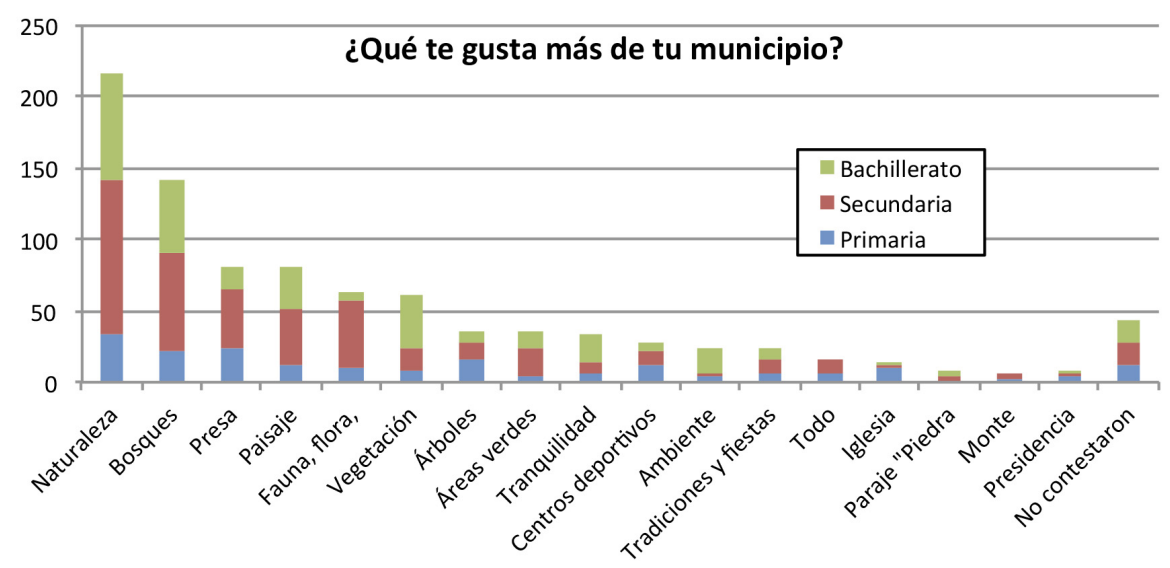

FIgURA 5. Gráfico de respuestas más frecuentes

del municipio; también mencionaron recurrentemente "el paisaje”, "los árboles”, "la vegetación”, "las áreas verdes” y "la tranquilidad”. De esto se desprende que es claro que los niños reconocen que viven en una zona rural y aprecian las características asociadas con ese entorno natural, poco urbanizado.

En cuanto a lo que menos les gusta de su municipio, los niños identificaron "la contaminación" en primer lugar, "la basura” en segundo y "la tala" en tercero, reconociendo así aquellos factores que disminuyen la calidad del entorno 
Participación social y educación ambiental para la conservación. Un estudio de caso con niños y jóvenes de una zona rural periurbana

natural (figura 6). Otras respuestas dentro de lo que menos les gusta incluyeron características que comprometen la comodidad, por ejemplo "el frío", y aspectos sociales como "pocos servicios" (principalmente entre los alumnos de bachillerato), "la delincuencia” y "la gente cerrada”, refiriéndose a la forma de socializar de la población local. Entre lo que harían para cuidar el monte los encuestados señalaron "no talar" como la principal solución, seguida de "no tirar basura” y "reforestar".

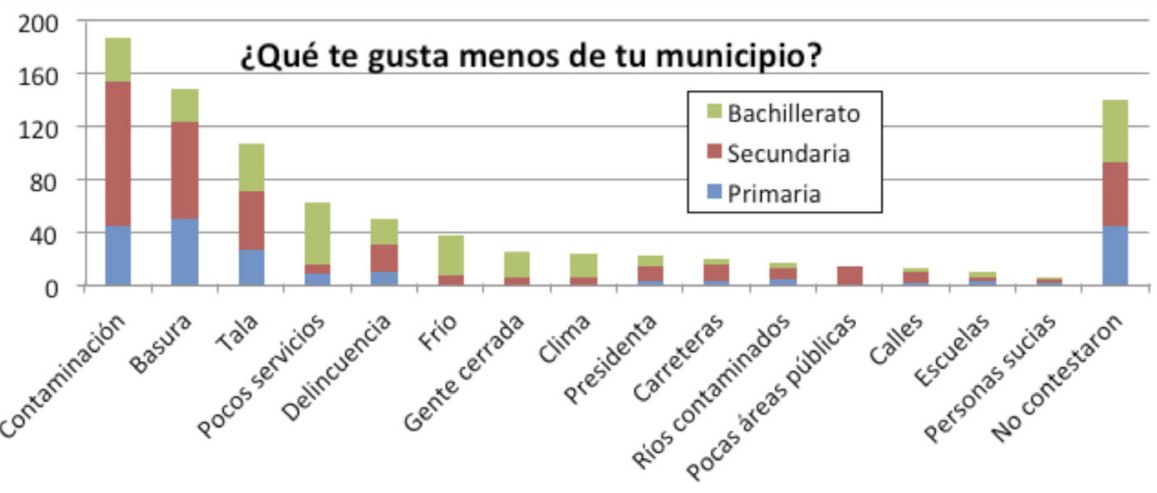

Figura 6. Gráfico de respuestas más frecuentes

Siendo los bosques el elemento principal que distingue el paisaje natural local, su uso y manejo resultan un tema sensible entre los pobladores. A pesar de que la mayoría de los bosques de la zona se encuentran en un área protegida en el nivel estatal (el corredor Otomí-Mexica), los habitantes reportan que la tala ilegal todavía sucede con frecuencia. Por otro lado, algunas zonas comunales de Isidro Fabela y Jilotzingo cuentan con un permiso de aprovechamiento forestal debidamente requisitado y verificado por Probosque (la autoridad forestal del Estado de México), de acuerdo con la reglamentación vigente. Sin embargo, la sociedad local dista mucho de percibir tal actividad como legítima, debido, según lo expresaron, a "eventos ocurridos en el pasado", cuando se talaban más árboles de lo permitido; a la presencia de técnicos forestales que no están debidamente capacitados o que no son totalmente honestos; a la impresión de que los beneficios económicos no se reparten de manera justa y equitativa entre 
todos los integrantes correspondientes, y a que no se tiene un plan adecuado de reforestación y restauración a largo plazo.

Otro tema sensible es el del agua que se consume en los municipios de Isidro Fabela y Jilotzingo, que proviene de manantiales en la parte alta, entre los bosques de pino y oyamel. El control de calidad del líquido y su distribución entre todos los pobladores es responsabilidad del ayuntamiento y los núcleos agrarios. Los habitantes deben pagar una cuota de alrededor de 200 pesos mexicanos por año (unos 12 usD, al tipo de cambio de noviembre de 2015), lo que solo realiza $30 \%$ de la población. Ante la pregunta del cuidado y buen uso del agua, los niños respondieron que no se debe desperdiciar, ni contaminar, ni tirar basura en los ríos. Muy pocos mencionaron que era importante pagar el servicio del agua, lo que pone de manifiesto que hay desconfianza ante el ejercicio que hace la autoridad en el nivel municipal y en otros niveles. La gente no reconoce la obligación de pagar por el agua, pues argumenta que el líquido es un recurso gratuito y que fueron sus familiares los que en otra época "realizaron faenas para traer el agua al pueblo a cambio de que no la cobraran”. La mayoría de los niños identificó una fuerte conexión entre la existencia del bosque y la provisión de agua, ya que a la pregunta iy qué tienen que ver los bosques con el agua? durante las charlas, casi siempre decían que "los árboles necesitan agua" y que "el bosque nos da agua".

En relación con el cuidado de la fauna, se reconocen como primeras acciones al respecto "no cazarlos", "no maltratarlos" y, en general, "cuidarlos". La diversidad faunística de la zona es importante pero ha disminuido drásticamente en los últimos años. Existen animales erróneamente considerados venenosos, por ejemplo el lagarto escorpión (Barisia imbricata), y por esta razón los pobladores locales los matan con facilidad. Durante las charlas se insistió en que esto no es así. El único animal venenoso de la región es la víbora de cascabel (Crotalus triseriatus). En opinión de la mayoría de los encuestados, la flora y los árboles "deben ser cuidados", y consideran necesario "no talarlos", "no maltratarlos" y "hacer reforestación”. Otras respuestas fueron "evitar incendios”, "regar los árboles” y "no usar plaguicidas".

Por último se les cuestionó cómo imaginaban y cómo querían que fuera su municipio en un futuro. A esto la mayoría ( $>75 \%$ ) contestó que le gustaría que estuviera conservado, limpio, sin contaminación, igual que ahora, pero 
Participación social y educación ambiental para la conservación. Un estudio de caso con niños y jóvenes de una zona rural periurbana

más seguro, lo que sugiere que también en esta región se percibe un aumento de la inseguridad y probablemente la reducción de la calidad de los servicios ambientales.

Entre los concursos de 2013, 2014 y 2015 se presentaron aproximadamente 350 carteles, elaborados en equipos de uno a cinco integrantes, por los niños y jóvenes de las primarias, secundarias y preparatorias de Isidro Fabela y Jilotzingo, en un tamaño no mayor al de una cartulina. Cada cartel tuvo oportunidad de ser exhibido de forma individual ante el jurado, que evaluó cada uno con base en tres aspectos: idea original, contenido y presentación, y así emitió calificaciones conjuntas en escala del 1 al 10, las cuales fueron capturadas en una base de datos en Excel para obtener promedios. Los mayores puntajes determinaron a los ganadores de cada categoría. La figura 7 muestra los diseños de algunos de los carteles ganadores para las categorías de primaria, secundaria y preparatoria.

Los carteles fueron muy coloridos, algunos con objetos colgantes, con materiales del bosque como hojas y ramas, restos de basura, etc. Muchos aludían a la biodiversidad de la región, con especial atención en los hongos, los árboles, los reptiles, las aves y los mamíferos. Varios incluyeron frases relacionadas con el medio ambiente, entre las que destacan: "Es increíble que la naturaleza pida a gritos ayuda y que nadie la escuche", "Isidro Fabela, cuídalo, no esperemos que sea demasiado tarde, reforestemos", "El ambiente nos da vida, por eso cuídalo y consérvalo bonito", "Tlazala es como una mariposa, gracias a tu cuidado, más hermosa", "El cuidado de la naturaleza es responsabilidad de todos", "El inconsciente destruye, el inteligente cuida, el sabio conserva; y tú, ¿qué quieres en tu monte?", "Lo que es contrario a la naturaleza no es bello y la naturaleza de Jilotzingo es muy bella”, "Los árboles son la llave de la vida para Jilotzingo, ¡cuídalos!”, “Mucha gente pequeña, en lugares pequeños, haciendo cosas pequeñas, pueden cambiar el mundo”.

Como premio, a todos los ganadores se les regalaron gorras con dibujo y mensaje bordados, así como ropa y balones deportivos. A los primeros lugares de cada categoría se les llevó a un parque de diversiones en la Ciudad de México con todos los gastos pagados (figura 8). 

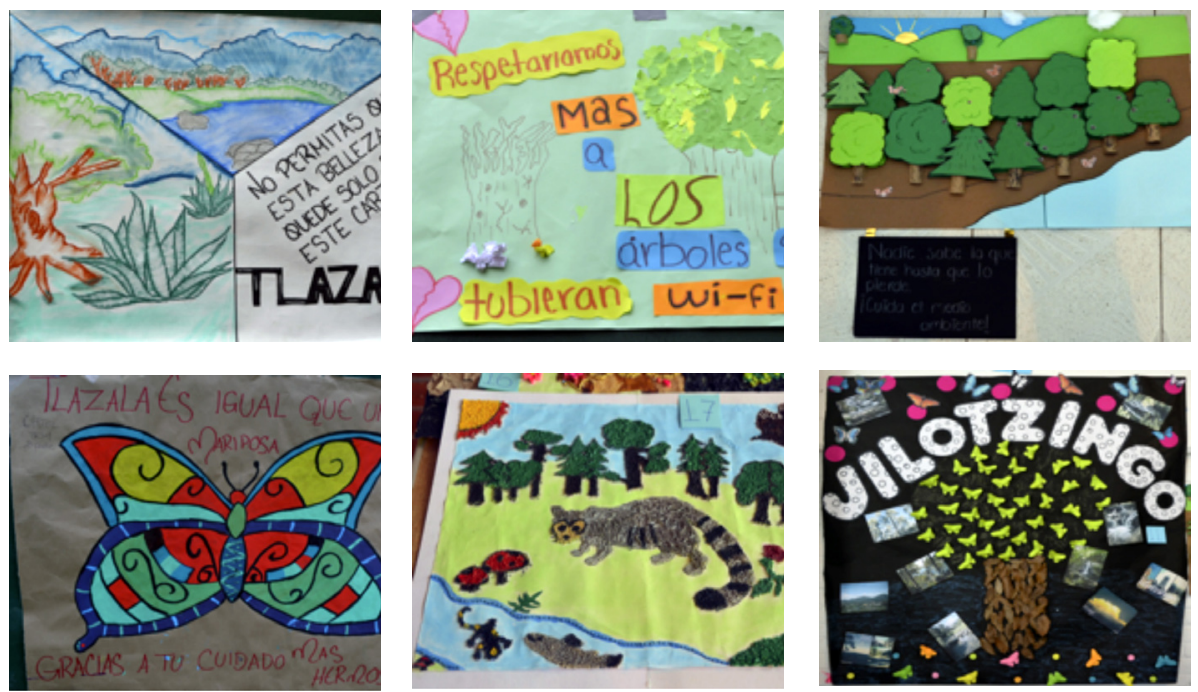

FigURA 7. Ejemplos de carteles en Isidro Fabela (2013 y 2014) y Jilotzingo (2015)
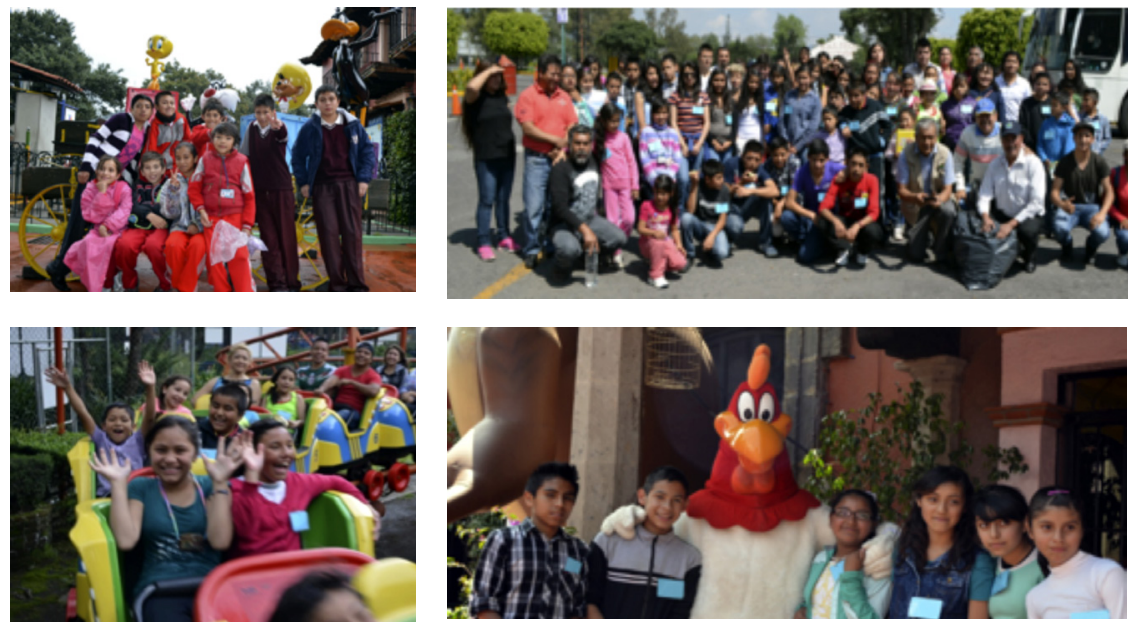

FiguRA 8. Equipos ganadores del concurso de carteles 2013, 2014 y 2015 
Participación social y educación ambiental para la conservación. Un estudio de caso con niños y jóvenes de una zona rural periurbana

\section{Conclusión}

Desde sus inicios como enfoque educativo, la educación ambiental ha promovido la toma de conciencia sobre el valor que se le da a la naturaleza con la finalidad de propiciar la participación social en actividades que prevengan y resuelvan la problemática ambiental (Barahona y Almeida-Leñero, 2006). Como se pudo constatar en las encuestas, los niños y jóvenes de esta región muestran un claro interés por el entorno natural en el que habitan, lo que se evidencia al manifestar que lo que más les gusta es la naturaleza del lugar, que desean que se conserve esta parte para el futuro, y que no quieren ver a su comunidad como la parte urbana de la Ciudad de México. También demostraron conocimiento de la problemática ambiental al sugerir no continuar con la tala para el cuidado y conservación del monte. Empero, aún son pocos los que alcanzan a percibir que las acciones individuales pueden influir seriamente en la conservación o deterioro del ambiente.

Entre los factores de deterioro ambiental más mencionados estuvieron la presencia recurrente de basura en todos los ambientes de ambos municipios (desde calles y lugares públicos, hasta el bosque y los cuerpos de agua) y la contaminación de los ríos. Sin embargo, no parece que les sea clara la cantidad de contaminantes que ellos mismos o sus familias generan ni las complicaciones que implican en sus municipios el manejo de la basura, la limpieza de los ríos y la provisión de servicios básicos para la población creciente. Por otro lado, se detectó que la mayoría de los niños y jóvenes entrevistados no se dan cuenta de que muchos de los bienes que utilizan provienen de la transformación de los recursos del bosque, como algunos muebles en su hogar hechos de madera, material de construcción (madera, arena, grava), el empleo de leña para preparar alimentos, o la provisión de una red de agua potable que llegue hasta la llave de su casa. Por tanto, para futuras experiencias será necesario buscar que la audiencia pase de una fase superficial de crítica de "los otros, los que se acaban el bosque”, hacia una concientización verdadera donde se demuestre que reconocen que la solución está en ellos. Si bien en tres años se ha notado un cambio de actitud, aún hay mucho por hacer. Sería importante, por ejemplo, reforzar el mensaje a lo largo del año con pequeñas acciones coordinadas.

El uso de actividades recreativas, como los concursos de carteles y la entrega de premios a los ganadores, hace posible el acercamiento directo con los 
niños y jóvenes, e indirectamente con los adultos a través de las familias de los concursantes, profesores, funcionarios y otras personas clave en la comunidad. La integración de los niños y jóvenes a temprana edad en temas ambientales les permite tener conocimiento fundamental para la toma de decisiones futuras (Robles, 2011). Esperamos que la transmisión del conocimiento en las pláticas sobre la biodiversidad local, servicios ambientales y su aprovechamiento apropiado fomente despertar la conciencia crítica de los habitantes más jóvenes e incluso contagiar a los adultos a darse cuenta de la riqueza natural del ambiente en el que viven. Si bien no hay tiempo para esperar a que los niños de hoy tomen el mando, exponer a las autoridades del lugar con los niños y jóvenes permitió iniciar un diálogo y promovió la adopción de compromisos morales en la toma de decisiones para cuestiones ambientales, lo que marca un paso cardinal en el ejercicio de la participación social y la comprensión de que la mejor solución a los daños ambientales está en prevenirlos desde ahora.

El trabajo de la educación ambiental no debe terminar aquí; resulta fundamental que sea un proceso continuo a corto, mediano y largo plazo en el que se impulse la difusión del conocimiento en los municipios estudiados, así como en otros municipios vecinos, con la implementación de nuevas técnicas para transferir información, y fomentar el interés y la participación activa de los habitantes en el buen uso y cuidado del ambiente. Esperamos que esto ayude a despertar una conciencia colectiva sobre el valor de conservar la naturaleza y sobre la relevancia de informarse y prepararse mejor para poder aprovechar los recursos naturales de forma moderada y con posibilidades de preservación para las generaciones futuras.

\section{Fuentes consultadas}

Barahona, A. y L. Almeida-Leñero (coords.). (2006). Educación para la conservación. México: Universidad Nacional Autónoma de México-Facultad de Ciencias-Coordinación de Servicios Editoriales.

Bravo Mercado, M., M. Arias Ortega, L. Barraza, V. Bedoy Velázquez, C. Gómez Luna, E. González Gaudiano, O. Pérez Peña, J. Reyes Ruiz y C. Velasco Samperio (2008). "La educación ambiental en México: visiones y 
- Participación social y educación ambiental para la conservación. Un estudio de caso con niños y jóvenes de una zona rural periurbana

proyecciones de actualidad”, en F. Reyes Escutia y M. T. Bravo Mercado (coords.). Educación ambiental para la sustentabilidad en México. Aproximaciones conceptuales, metodológicas y prácticas. Tuxtla Gutiérrez: Universidad de Ciencias y Artes de Chiapas (colección Selva Negra), 14-45.

Camarena-Gómez, B. O. (2006). "La educación ambiental en el marco de los foros internacionales: una alternativa de desarrollo". Estudios Sociales, 15 (28), 8-42.

Conagua y сCPG (2008). Programa Hídrico de Gran Visión de la Cuenca Presa Guadalupe [en línea]. México: Comisión Nacional del Agua/Comisión de Cuenca Presa Guadalupe. Disponible en: http://cuencapresaguadalupe.org/documentacion/programa-h\% C3\% ADdrico.html [2014, 19 de febrero].

Robles, M. (2011). “Dime qué ves y te diré qué piensas. El mundo de las percepciones y los retos para la comunicación ambiental”. Investigación Ambiental. Ciencia y Política Pública, 3 (1), 48-56.

Sarukhán, J. (2006). "Prefacio”, en A. Barahona y L. Almeida-Leñero (coords.). Educación para la conservación. México: Universidad Nacional Autónoma de México-Facultad de Ciencias-Coordinación de Servicios Editoriales, vii-xi.

Vega, P. y P. Álvarez (2005). "Planteamiento de un marco teórico de la educación ambiental para un desarrollo sostenible”. Revista Electrónica de Enseñanza de las Ciencias, 4 (1), 1-16. 